\section{Le maximum de la partie imaginaire des fonctions univalentes bornées}

\author{
par W. JANOWsKI (Eódź)
}

Dans ce travail nous obtenons certains résultats concernant le maximum de la partie imaginaire des fonctions univalentes bornées.

Ce travail appartient au cycle de travaux dont les résultats sont de la catégorie des théorèmes sur la déformation.

1. Considérons les fonctions holomorphes univalentes dans le cercle $|z|<1$ de la forme

$$
F(z)=z+A_{2} z^{2}+\ldots
$$

Soit $M>1$ un nombre positif quelconque, $F_{M}$ - la famille de toutes les fonctions bornées de la forme (1) assujetties à la condition $|F(z)|<M$, et $F_{\infty}$ - la famille de toutes les fonctions de la forme (1).

La valeur $1>r>0$ étant fixée, pour chaque $F(z)$ nous considérons

$$
I\{F(r)\} \text {. }
$$

Ainsi formée, l'expression (2) est une fonctionnelle définie dans la famille $F_{\infty}$ et par conséquent dans $F_{M}$.

THÉoRÈme 1. Pour les fonctions de la famille $F_{M}$ on a l'inégalité

$$
I\{F(r)\} \leqslant \varrho_{M} \sin \varphi_{M},
$$

où $\varrho_{M}\left(0<\varrho_{M}<r\right)$ et $\varphi_{M}\left(\sin \varphi_{M}>0\right)$ présentent une solution des équations

$$
\frac{\left[1+(\varrho / M)^{2}\right] \sin \varphi-2 \varrho / M}{(2 \varrho / M) \sin \varphi-\left[1+(\varrho / M)^{2}\right]} \log \left(\frac{1-\varrho / M}{1+\varrho / M}: \frac{1-r}{1+r}\right)+
$$

$$
+\log M \frac{1-r^{2}}{r(M L / \varrho-\varrho / M)}=0
$$

$$
\varphi=\left[\log ^{2}\left(\frac{1-\varrho / M}{1+\varrho / M}: \frac{1-r}{1+r}\right)-\log ^{2} M \frac{1-r^{2}}{r(M / \varrho-\varrho / M)}\right]^{1 / 2},
$$

telle que le produit $\varrho \sin \varphi$ est le plus grand.

La limite (3) est atteinte.
Théorł̀me 2. Pour les fonctions de la famille $F_{\infty}$ on a l'inégalité

$$
I\{F(r)\} \leqslant \varrho_{\infty} \sin \varphi_{\infty},
$$

ò̀ $\varrho_{\infty}$ et $\varphi_{\infty}$ satisfont aux équations

$$
\log \varrho_{\infty}=\log \left(r /\left(1-r^{2}\right)\right)+\sin \varphi_{\infty} \log ((1+r) /(1-r))
$$

$$
\varphi_{\infty}=\cos \varphi_{\infty} \log ((1+r) /(1-r))
$$

et cette limite est atteinte.

Avant de procéder à la démonstration nous remarquons que nos théorèmes sont équivalentes à ceux qui établissent l'existence de la fonction extrémale par rapport à la fonctionnele (2) dans la famille $F_{M}$, resp. dans $F_{\infty}$. La partie imaginaire de cette fonction est égale à $\varrho_{M} \sin \varphi_{M}$ resp. $\varrho_{\infty} \sin \varphi_{\infty}$, où $\varrho_{M}$ et $\varphi_{M}$ sont des racines des équations (4), resp. $\varrho_{\infty}$ et $\varphi_{\infty}-$ des équations (5).

Démonstration du théorème 1. Pour démontrer l'avant dérnier théorème considérons d'abord la famille $F_{T}{ }^{1}$ ) de toutes les fonctions univalentes bornées dans $|z|<1$ de la forme

$$
f(z)=a_{1} z+a_{2} z^{2}+\ldots=a_{1}\left(z+A_{2} z^{2}+\ldots\right),
$$

où $a_{1} \geqslant T, T$ étant un nombre quelconque fixe de l'intervalle $(0,1)$. Ces fonctions sont assujetties à la condition $|f(z)|<1$ pour tout $|z|<1$. Considérons encore la fonctionnelle qui y est déterminée,

$$
K(f)=I\left\{f(r) / a_{1}\right\} .
$$

Nous constatons que la fonctionnelle (6) a dans chaque point $f$ de la famille $F_{T}$ une différentielle qui ne s'annule identiquement dans aucun point de $F_{T}$. En effet, en désignant par $\Delta f=\Delta f(z)=\Delta a_{1} z+\Delta a_{2} z^{2}+\ldots$ la fonction quelconque, telle que $f(z)+\Delta f(z) \in F_{T}$, on a

(7) $\quad K(f+\Delta f)=I\left\{(f(r)+\Delta f(r)) /\left(a_{1}+\Delta a_{1}\right)\right\}$

$$
=I\left\{f(r) / a_{1}\right\}+I\left\{\Delta f(r) / a_{1}\right\}-I\left\{f(r) \Delta a_{1} / a_{1}^{2}\right\}+\eta(\Delta f),
$$

$$
L(h)=I\left\{h(r) / a_{1}\right\}-I\left\{f(r) a_{1 h} / a_{1}^{2}\right\} \quad \text { pour chaque } h \in H
$$

(voir [3], p. 43) est une fonctionnelle linéaire et

$$
\eta(\Delta f)<\left([\Delta f(r)]^{2}+\left(\Delta a_{1}\right)^{2}\right)^{1 / 2} P\left(\Delta f(r), \Delta a_{1}\right),
$$

où $P(\xi, \eta) \rightarrow 0$ pour $\xi \rightarrow 0, \eta \rightarrow 0$. Comme

(10) $|\Delta f(r)|<\|\Delta f\|_{n} \quad$ pour $\quad(1-1 / n)^{-1}>r, \quad\left|\Delta a_{1}\right|<(1-1 / n)^{-1}\|f(z)\|_{n}$

1) La famille introduit par M. Z. Charzyński, comparer [2], p. 5. 
pour $n$ assez grand, selon l'inégalité de Cauchy, on a de (9)-(10), en posant $\left(1+(1-1 / n)^{-2}\right)^{1 / 2} P\left(\Delta f, \Delta a_{1}\right)=\varepsilon(\Delta f)$, que

$$
\eta(\Delta f)<\|f(z)\|_{n} \varepsilon(\Delta f(z)),
$$

ce qui prouve (vu (11 et (7)) que la fonctionnelle (6) est différenciable. dans $F_{T}$ et que la différentielle de (6) au point $f$ est donnée par (8). En outre, il résulte de (8) que $\mathrm{L}(h)$ ne s'annule pas identiquement dans aucun point de $F_{T}$.

On voit aisément que la différentielle (8), prise en un point quelconque de la famille $F_{T}$, s'étend sur la famille linéaire $\mathfrak{B}$ de toutes les fonctions méromorphes dans le cercle $K(0,1)$, possédant des pôles dans l'ensemble $E^{*}$ composé des points appartenant à ce cercle et différents de $r$. La frontière de l'ensemble $E^{*}$ contient la circonférence $K^{*}(0,1)$, dont aucun point n'est un point d'accumulation des points essentiels (voir $[2]$, p..25) de l'ensemble complémentaire de $E^{*}$.

On peut donc appliquer à la fonctionnelle $K(f)$ les résultats de $\mathrm{Z}$. Charzyński et W. Janowski (voir [3], p. 45) relatifs aux fonctions extrémales par rapport à la fonctionnelle $K(f)$ dans la famille $F_{T}$.

Il résulte de ces considérations qu'il existe des fonctions extrémales respectives et qu'elles satisfont à l'équation

$$
\left[f^{* \prime}(z) / f^{*}(z)\right] \mathscr{N}\left[f^{* \prime}(z)\right]=z^{-2} \Re(z) \quad \text { pour } \quad z \in E^{*}
$$

(voir $[3]$, p. 53), où

$$
\begin{aligned}
\Re(\omega) & \equiv D^{*}\left[\Psi\left(f^{*}(\zeta), \omega^{-1}\right)\right]+\hat{D}^{*}\left[\Psi\left(\hat{f}^{*}(\zeta), \omega\right)\right]-2 P^{*}, \\
\Re(z) & \equiv D^{*}\left[\Psi(\zeta, 1 / z) f^{* \prime}(\zeta)\right]+\hat{D}^{*}\left[\Psi(\zeta, z) \hat{f}^{* \prime}(\zeta)\right]-2 P^{*} .
\end{aligned}
$$

$\zeta$ désigne la variable apparente de l'opération tandis que $z$ joue dans (13) le rôle d'un paramètre.

$$
\begin{aligned}
\psi(\omega, \lambda) & \equiv \omega(1+\omega \lambda) /(1-\omega \lambda), \\
D^{*}(h) & =L^{*}(h)-i L^{*}(h i), \\
\hat{D}^{*}(\hat{h}) & =L^{*}(h)+i L^{*}(h i)
\end{aligned}
$$

(voir [3], p. 43). $L^{*}(h)$ est la différentielle de la fonctionnelle (6) au point $f^{*}$.

D'après (14)

$$
\mathfrak{P}^{*}=\min _{0 \leqslant y<2 \pi}\left\{D^{*}\left[\psi\left(f^{*}(\zeta), e^{-i \nu}\right)\right]+\hat{D}^{*}\left[\psi\left(\hat{f}^{*}(\zeta), e^{i \nu}\right)\right]\right\} / 2 .
$$

$$
D^{*}(h)+\hat{D}^{*}(\hat{h})=2 L^{*}(h)
$$

$\mathrm{Vu}$ (15) et: (8), les formules (13) prendront la forme:

$$
\begin{aligned}
\mathfrak{N}(\omega) & \equiv 2 I\left\{\frac{\psi\left[f^{*}(r), 1 / \omega\right]}{a_{1}^{*}}-\frac{f^{*}(r)}{a_{1}^{*}}\right\}-2 \mathfrak{P}^{*} \\
& =\frac{1}{a_{1}^{*} i}\left\{\psi\left[f^{*}(r), 1 / \omega\right]-\overline{\psi\left[f^{*}(r), 1 / \omega\right]}-\left(f^{*}(r)-\overline{f^{*}(r)}\right)\right\}-2 \mathfrak{P}^{*}, \\
\mathcal{Y}(z) & \equiv 2 I\left\{\frac{\psi(r, 1 / z) f^{* \prime}(r)}{a_{1}^{*}}-\frac{f^{*}(r)}{a_{1}^{*}}\right\}-2 \mathfrak{P}^{*} \\
& =\frac{1}{a_{1}^{*} i}\left\{\psi(r, 1 / z) f^{* \prime}(r)-\overline{\psi(r, 1 / z) f^{* \prime}(r)}-\left(f^{*}(r)-\overline{f^{*}(r)}\right)\right\}-2 \mathfrak{P}^{*},
\end{aligned}
$$

d'où, ell posant successivement dans (14) $\omega=f^{*}(r), \lambda=1 / \omega$ et $\omega=r, \lambda=1 / z$, on a, d'après $(16)$ et après des calculs faciles:

(17)

$$
\begin{aligned}
\mathfrak{R}_{(}(\omega) & \equiv \frac{1}{a_{1}^{*} i}\left[\frac{\omega+f^{*}(r)}{\omega-f^{*}(r)} f^{*}(r)-\frac{1+\overline{f^{*}(r) \omega}}{1-\overline{f^{*}(r)} \omega} \overline{f^{*}(r)}-\left(f^{*}(r)-f^{*}(r)\right)\right]-2 \mathfrak{P}^{*}, \\
\text { (18) } \Re(z) & \equiv \frac{1}{a_{1}^{*} i}\left[r f^{* \prime}(r) \frac{z+r}{z-r}-r f^{* \prime}(r) \frac{1+r z}{1-r z}-\left(f^{*}(r)-\overline{\left.f^{*}(r)\right)}\right]-2 \mathfrak{P}^{*} .\right.
\end{aligned}
$$

Posons maintenant pour abréger

$$
f^{*}(z)=\omega, \quad f^{* \prime}(z)=\omega^{\prime},
$$

et soit

$$
\begin{aligned}
f^{*}(r)=\varrho e^{i \varphi}, \quad \overline{f^{*}}(r) & =\varrho e^{-i \phi}, \quad f^{* \prime}(r)=\tau e^{i \theta}, \quad \overline{f^{* \prime}(r)}=\tau e^{-i \theta}, \\
\mathfrak{P}^{*} & \left.=(i a-\varrho \sin \varphi) / a_{1}^{* 2}\right) .
\end{aligned}
$$

En multipliant les deux membres par $a_{1}^{*} i$ et vu (17), (18), (19) et (20) l'équation (12) prendra alors la forme suivante:

$$
\begin{gathered}
\frac{\omega^{\prime 2}}{\omega^{2}}\left(\varrho e^{i \varphi} \frac{\omega+\varrho e^{i \varphi}}{\omega-\varrho e^{i \varphi}}-\varrho e^{-i \varphi} \frac{1+\varrho e^{-i \varphi} \omega}{1-\varrho e^{-i \varphi} \omega}+2 \alpha\right) \\
=\frac{1}{z^{2}}\left(r \tau e^{i \theta} \frac{z+r}{z-r}-r \tau e^{-i \theta} \frac{1+r z}{1-r z}+2 \alpha\right) .
\end{gathered}
$$

L'équation que nous avons obtenue peut être écrite sous une forme plus simple en vertu de certaines propriétés des expressions (17) et (18) que nous allons examiner maintenant. $\mathfrak{N}(\omega)$ et $\Re(z)$, comme il a été dé

2) $\mathrm{P}^{*} \leqslant 0$; comparer [3], p. 48. De là $I\{a\} \geqslant 0$. Nous utilisérons cette remarque plus loin. 
montré dans le travail cité (voir [3], p. 46), prennent sur la circonférence du cercle $|\omega|=1$ (respectivement $|z|=1$ ) des valeurs réelles non négatives. $\mathfrak{M}(\omega)=0$ ayant, comme cela résulte de la définition de $\mathfrak{P}^{*}$, une racine double sur la circonférence du cercle $|\omega|=1$, n'a pas d'autres racines, ni à l'intérieur du cercle $|\omega|=1$, ni sur sa circonférence, puisqu'elle n'a que deux racines. C'est ce qui résulte directement de la forme $\mathfrak{M}(\omega)$ (formule (17)).

$\Re(z)$ a deux racines. C'est ce qui résulte aussi directement de la forme de $\Re(z)$ (formule (18)).

$\Re(z)$ ne peut pas posséder de racine à l'intérieur du cercle $|z|<1$, car alors, en vertu de $(12)$ et vu $f^{* \prime}(z) \neq=0, \mathfrak{N}(\omega)=0$ aurait une racine à l'intérieur du cercle $|\omega|<1$, ce qui serait contraire à ce que nous venons de démontrer. Cella posé, $\Re(z)=0$ n'a de racines que sur la circonférence du cercle $|z|=1$. Comme la fonction $\Re(z)$ n'est pas négative sur la circonférence $|z|=1, \mathfrak{N}(z)=0$ ne posséde qu'une seule racine double.

Il s'ensuit de ce que nous venons de démontrer qu'il existe des nombres $p_{i} ; q_{i}(i=1,2)$, différents de zéro et tels que les expressions contenues entre les parenthèses de l'équation (21) prennent respectivement les formes suivantes:

$$
\varrho e^{i \varphi} \frac{\omega+\varrho e^{i q}}{\omega-\varrho e^{i \varphi}}-\varrho e^{-i_{\varphi}} \frac{1+\varrho e^{-i \varphi} \omega}{1-\varrho e^{-i \varphi} \omega}+2 \alpha=\frac{\left(p_{1} \omega-q_{1}\right)^{2}}{\left(\omega-\varrho e^{i \eta}\right)\left(1-\varrho e^{-i \phi} \omega\right)},
$$

$$
r \tau e^{i \theta} \frac{z+r}{z-r}-r \tau e^{-i \Downarrow} \frac{1+r z}{1-r z}+2 \alpha \equiv \frac{\left(p_{2} z-q_{2}\right)^{2}}{(z-r)(1-r z)} .
$$

Par suite l'équation (21) prendra la forme

$$
\frac{\omega^{\prime 2}}{\omega^{2}} \cdot \frac{\left(p_{1} \omega-q_{1}\right)^{2}}{\left(\omega-\varrho e^{i \varphi}\right)\left(1-\varrho e^{-i \phi} \omega\right)}=\frac{1}{z^{2}} \cdot \frac{\left(p_{2} z-q_{2}\right)^{2}}{(z-r)(1-r z)} .
$$

En posant dans l'équation (23)

$$
q_{1} / p_{1}=\omega_{0}, \quad q_{2} / p_{2}=z_{0}
$$

et en divisant les deux membres de l'équation (23) par $p_{1}^{2}$, puis en po$\operatorname{sant}$

$$
p_{2}^{2} / p_{1}^{2}=t
$$

nous obtenons l'équation

$$
\frac{\omega^{\prime 2}}{\omega^{2}} \cdot \frac{\left(\omega-\omega_{0}\right)^{2}}{\left(\omega-\varrho e^{i \varphi}\right)\left(1-\varrho e^{-i \varphi} \omega\right)}=\frac{t}{z^{2}} \cdot \frac{\left(z-z_{0}\right)^{2}}{(z-r)(1-r z)} .
$$

Posons maintenant

$$
\begin{gathered}
\left(\varrho e^{i \varphi}\right)^{1 / 2}=\varrho^{1 / 2} e^{i q / 2}, \quad\left(e^{-2 i \varphi}\right)^{1 / 2}=e^{-i_{\varphi}}, \quad\left(-\varrho e^{i \varphi}\right)^{1 / 2}=i\left(\varrho e^{i q}\right)^{1 / 2}, \\
\left(-\varrho e^{-i \varphi}\right)^{1 / 2}=i\left(\varrho e^{-i \varphi}\right)^{1 / 2}=i \varrho^{1 / 2} e^{-i_{\varphi / 2}}, \quad(-r)^{1 / 2}=i r^{1 / 2}
\end{gathered}
$$

De (26) on obtient.

$$
\omega^{\prime} \cdot \frac{\omega-\omega_{0}}{\left[\left(\omega-\varrho e^{i \phi}\right)\left(1-\varrho e^{-i \varphi} \omega\right)\right]^{1 / 2}}=\frac{t^{1 / 2}}{z} \cdot \frac{z-z_{0}}{[(z-r)(1-r z)]^{1 / 2}},
$$

où l'on a pris les branches des radicaux $[(z-r)(1-r z)]^{1 / 2},\left[\left(\omega-\varrho e^{i \varphi}\right) \times\right.$ $\left.\times\left(1-\varrho e^{-i q} \omega\right)\right]^{1 / 2}$, qui sont égales (pour $z=0$ et $\omega=0$ ) $\grave{a} i r^{1 / 2}$, resp. $i\left(\varrho e^{i q}\right)^{1 / 2}$.

Ces branches existent respectivement dans le cercle $K(0, r)$ et dans le domaine $\omega[K(0, r)]$, où les expressions sous les signes des radicaux sont différentes de zéro. Puisque $\omega(0)=0$, il s'ensuit de (28) que

$$
t^{1 / 2}=\left(\omega_{0} / z_{0}\right)\left(r^{1 / 2} / \varrho^{1 / 2}\right) e^{-i \varphi / 2} .
$$

En intégrant les deux membres de cette équation nous obtiendrons, après des calculs aisés et vu (29):

$$
\begin{aligned}
& \text { (30) } \log \frac{\varrho e^{-i \varphi} \omega-1+\left[\left(\varrho e^{-i \varphi} \omega-1\right)\left(\varrho e^{-i \varphi} \omega-\varrho^{2}\right)\right]^{1 / 2}}{\varrho e^{-i \varphi} \omega-1-\left[\left(\varrho e^{-i \varphi} \omega-1\right)\left(\varrho e^{-i \varphi} \omega-\varrho^{2}\right)\right]^{1 / 2}}+ \\
& +\omega_{0} e^{-i \varphi} \log \frac{\varrho e^{i \varphi}\left(1-\varrho e^{-i \varphi} \omega\right)+\left[\varrho e^{i \varphi}\left(1-\varrho e^{-i \varphi} \omega\right)\left(\varrho e^{i \varphi}-\omega\right)\right]^{1 / 2}}{\varrho e^{i \varphi}\left(1-\varrho e^{-i \varphi} \omega\right)-\left[\varrho e^{i \varphi}\left(1-\varrho e^{-i \varphi} \omega\right)\left(\varrho e^{i \varphi}-\omega\right)\right]^{1 / 2}} \\
& =\frac{\omega_{0}}{z_{0}} e^{-i \varphi}\left[\log \frac{r z-1+[r(r z-1)(z-r)]^{1 / 2}}{r z-1-[r(r z-1)(z-r)]^{1 / 2}}+\right. \\
& \left.\left.+z_{0} \log \frac{r(1-r z)+[r(1-r z)(r-z)]^{1 / 2}}{r(1-r z)-[r(1-r z)(r-z)]^{1 / 2}}\right]+C^{3}\right)
\end{aligned}
$$

où l'on a pris les branches logarithmiques qui, pour $\omega=f^{*}(r)$ et $z=r$ respectivement, ont des valeurs principales. Ces branches existent dans les domaines simplement connexes $K(0, r)$ et $\omega[K(0, r)]$. En tenant compte de ce que $\omega(z) \rightarrow f^{*}(r)$, lorsque $z \rightarrow r$, nous obtenons immédiatement de (30) que $C=0$.

$$
\text { 3) } \begin{aligned}
& \int \frac{(x-a) d x}{x[(x-b)(1-c x)]}=(-c)^{-1 / 2} {\left[\log \frac{c x-1+[(c x-1)(c x-b c)]^{1 / 2}}{c x-1-[(c x-1)(c x-b c)]^{1 / 2}}+\right.} \\
&\left.+a\left(\frac{c}{b}\right)^{1 / 2} \log \frac{b(1-c x)+[b(1-c x)(b-x)]^{1 / 2}}{b(1-c x)-[b(1-c x)(b-x)]^{1 / 2}}\right] .
\end{aligned}
$$


Notre but consiste à déterminer le produit $\varrho \sin q$. Examinous dans ce but l'équation (30) dans l'entourage du point $z=0$. En développant les différentes expressions de (30) pour les $z$ suffisamment petits, nous obtenons les égalités suivantes:

$$
\begin{aligned}
& \left.(1)=T z+a_{2} z^{2}+\ldots=O_{1}(z)^{4}\right), \\
& {\left[\left(\varrho e^{-i \varphi} \omega-1\right)\left(\varrho e^{-i \varphi} \omega-\varrho^{2}\right]\right)^{1 / 2}=\varrho-2^{-1} e^{-i p}\left(1+\varrho^{2}\right) \omega+\ldots=\varrho+O_{2}(z) \text {, }} \\
& {\left[\varrho e^{i{ }^{i}}\left(1-\varrho e^{-i \varphi} \omega\right)\left(\varrho e^{i \varphi}-\omega\right)\right]^{1 / 2}=\varrho e^{i_{\varphi}}-2^{-1}\left(1+\varrho^{2}\right)(\omega+\ldots} \\
& =\varrho e^{i q}-2^{-1} T\left(1+\varrho^{2}\right) z+O_{3}\left(z^{2}\right),
\end{aligned}
$$

$\log \frac{\varrho e^{-i \varphi} \omega-1+\left[\left(\varrho e^{-i \varphi} \omega-1\right)\left(\varrho e^{-i \varphi} \omega-\varrho^{2}\right)\right]^{1 / 2}}{\varrho e^{-i \varphi} \omega-1-\left[\left(\varrho e^{-i \varphi} \omega-1\right)\left(\varrho e^{-i \varphi} \omega-\varrho^{2}\right)\right]^{1 / 2}}$

(31)

$$
=\log \left[(1-\varrho)(1+\varrho)^{-1}\right]+e^{-i \varphi} \omega+\ldots=\log \left[(1-\varrho)(1+\varrho)^{-1}\right]+O_{4}(z),
$$$$
\log \frac{\varrho e^{i \varphi}\left(1-\varrho e^{-i \varphi} \omega\right)+\left[\varrho e^{i \varphi}\left(1-\varrho e^{-i \varphi} \omega\right)\left(\varrho e^{i \varphi}-\omega\right)\right]^{1 / 2}}{\varrho e^{i \varphi}\left(1-\varrho e^{-i \varphi} \omega\right)-\left[\varrho e^{i \varphi}\left(1-\varrho e^{-i \varphi} \omega\right)\left(\varrho e^{i \varphi}-\omega\right)\right]^{1 / 2}}
$$$$
=\log 2 \varrho e^{i \varphi}-\log \left[\left(1-\varrho^{2}\right) 2^{-1}\right]-\log \omega-\left[\left(1+3 \varrho^{2}\right)(4 \varrho)^{-1}\right] e^{-i p} \omega+\ldots
$$$$
=\log 2 \varrho e^{i \varphi_{p}}-\log \left[T\left(1-\varrho^{2}\right) 2^{-1}\right]-\log z+O_{5}(z)+2 k_{1} \pi i
$$

$$
\left(k_{1}=0, \pm 1, \ldots\right) \text {. }
$$

D'une manière analogue:

$$
\log \frac{r z-1+[r(r z-1)(z-r)]^{1 / 2}}{r z-1-[r(r z-1)(z-r)]^{1 / 2}}=\log \left[(1-r)(1+r)^{-1}\right]+O_{6}(z),
$$

$$
\log \frac{r(1-r z)+[r(1-r z)(r-z)]^{1 / 2}}{r(1-r z)-[r(1-r z)(r-z)]^{1 / 2}}
$$

$$
=\log 2 r-\log \left[\left(1-r^{2}\right) 2^{-1}\right]-\log z+O_{7}(z)+2 k_{2} \pi i \quad\left(k_{2}=0, \pm 1, \ldots\right),
$$

où $O_{i}\left(z^{k}\right)$ sont d'un rang non inférieur à $1(k=1,2 ; i=1,2, \ldots, 7)$. En substituant les expressions (31) et (32) dans (30), nous obtenons, en vertu de la définition de la fonction $O_{i}\left(z^{k}\right)$, l'équation:

(33) $\quad \log [(1-\varrho) /(1+\varrho)]+\omega_{0} e^{-i \varphi}\left\{\log 2 \varrho e^{i_{p}}-\right.$

$$
\left.-\log \left[T\left(1-\varrho^{2}\right) / 2\right]-\log z+2 k_{1} \pi i\right\}
$$

$=\left(\omega_{0} / z_{0}\right) e^{-i \varphi}\{\log [(1-r) /(1+r)]+$

$$
\left.+z_{0}\left(\log 2 r-\log \left[\left(1-r^{2}\right) / 2\right]-\log z+2 k_{2} \pi i\right)\right\}
$$

4) Le premiér coefficient du développenent de la fonction extrémale est égal à $T$, comparer [3], p. 52 . d'où, après une réduction aisée et après avoir divisé les deux membres par $\omega_{0} e^{-i \varphi}$, nous obtenons l'équation.

$$
\begin{aligned}
& \left(1 / \omega_{0}\right) e^{i \varphi} \log [(1-\varrho) /(1+\varrho)]+\log \varrho e^{i \varphi}-\log T\left(1-\varrho^{2}\right)+2 k \pi i \\
= & \left(1 / z_{0}\right) \log [(1-r) /(1+r)]+\log \left[r /\left(1-r^{2}\right)\right] \quad\left(k=k_{1}-k_{2}\right) .
\end{aligned}
$$

On obtient sans peine de l'équation (34) deux équations auxquelles satisfont $\varrho$ et $\varphi$; en comparant respectivement les parties réelles et imaginaires des deux membres de l'équation (34) on obtient

$$
\begin{gathered}
R\left\{\left(1 / \omega_{0}\right) e^{i \varphi}\right\} \log [(1-\varrho) /(1+\varrho)]+\log \left[\varrho / T\left(1-\varrho^{2}\right)\right] \\
=R\left\{1 / z_{0}\right\} \log [(1-r) /(1+r)]+\log \left[r /\left(1-r^{2}\right)\right],
\end{gathered}
$$

$I\left\{\left(1 / \omega_{0}\right) e^{i \varphi}\right\} \log [(1-\varrho) /(1+\varrho)]+\varphi+2 k \pi=I\left\{1 / z_{0}\right\} \log [(1-r) /(1+r)]$. (22)

Évaluons maintenant $\omega_{0}$ et $z_{0}$. Reprenons dans ce but les identités

Il résulte de $(22)$ en tenant compte de (24), et après des calculs faciles, que

$$
\text { (36) } \begin{aligned}
p_{1}^{2}\left(\omega-\omega_{0}\right)^{2} \equiv-2 & \left\{(\alpha+\varrho \cos \varphi) \varrho e^{-i \varphi} \omega^{2}-\right. \\
& \left.-\left[\left(i-\varrho^{2}\right) \varrho i \sin \varphi+\alpha\left(1+\varrho^{2}\right)\right] \omega+(\alpha-\varrho \cos \varphi) \varrho e^{i \varphi}\right\}, \\
p_{2}^{2}\left(z-z_{0}\right)^{2} \equiv-2 & \left\{(\alpha+\tau r \cos \vartheta) r z^{2}-\right. \\
& \left.-\left[\left(1-r^{2}\right) \tau r i \sin \vartheta+\alpha\left(1+r^{2}\right)\right] z+(\alpha-\tau r \cos \vartheta) r\right\} .
\end{aligned}
$$

En comparant les coefficients de $\omega$ dans les deux membres de l'identité (36) on a

$$
\omega_{0}=\left[\left(1-\varrho^{2}\right) \varrho i \sin \varphi+a\left(1+\varrho^{2}\right)\right][2(\alpha+\varrho \cos \varphi) \varrho]^{-1} e^{i \varphi} .
$$

On determine $\alpha$, en profitant du fait que le membre droit de (36) est un carré (car le membre gauche est un carré). On a ainsi

c'est-à-dire

$$
\left[\left(1-\varrho^{2}\right) \varrho i \sin \varphi+\alpha\left(1+\varrho^{2}\right)\right]^{2}=4\left(\alpha^{2}-\varrho^{2} \cos ^{2} \varphi\right) \varrho^{2}
$$

(39) $\quad\left(1-\varrho^{2}\right)^{2} \alpha^{2}+2 \alpha \varrho\left(1-\varrho^{4}\right) i \sin \varphi+4 \varrho^{4}-\varrho^{2}\left(1+\varrho^{2}\right)^{2} \sin ^{2} \varphi=0$.

En désignant par $a_{1}$ et $a_{2}$ les racines de l'équation (39), on a

$$
a_{12}=\left[\varrho i /\left(1-\varrho^{2}\right)\right]\left[-\left(1+\varrho^{2}\right) \sin \varphi \pm 2 \varrho\right] .
$$

Il résulte immédiatement de la définition de $\mathfrak{P}^{*}$, de $\alpha$ et de (21) que

$$
\begin{aligned}
-2 \alpha=\min _{0 \leqslant \nu<2 \pi}\left[\varrho e^{i \varphi}\left(e^{i \nu}+\varrho e^{i \varphi}\right)\left(e^{i \nu}-\varrho e^{i \varphi}\right)^{-1}-\right. & \\
& \left.-\varrho e^{-i \varphi}\left(1+\varrho e^{-i \varphi} e^{i \nu}\right)\left(1-\varrho e^{-i \varphi} e^{i \nu}\right)^{-1}\right] .
\end{aligned}
$$


Il faut donc prendre celle des racines $\alpha_{1}$ et $\alpha_{2}$ qui est la plus grande, e'est- . à-dire

$(40)$

$$
a=\left[\varrho i /\left(1-\varrho^{2}\right)\right]\left[-\left(1+\varrho^{2}\right) \sin \psi+2 \varrho\right] .
$$

Posons pour chaque $x$ et $y$,

$$
\begin{gathered}
A(x, y) \equiv\left(1+x^{2}\right) \sin y-2 x, \quad B(x, y) \equiv\left(1-x^{2}\right) \cos y, \\
O(x, y) \equiv 1+x^{2}-2 x \sin y .
\end{gathered}
$$

D'après $(40)$ et $(41)$ on a que $\alpha=\varrho i\left(\varrho^{2}-1\right)^{-1} A(\varrho, \varphi)$. En substituant $\alpha$ dans (38) nous obtenons

$$
\omega_{0}=-e^{i \varphi} C(\varrho, \varphi)[A(\varrho, \varphi)+i B(\varrho, \varphi)]^{-1} .
$$

Pour évaluer $z_{0}$ on remarque d'abord, qu'en vertu de (36) et (37),

$$
p_{1}^{2}=-2(\alpha+\varrho \cos \varphi) \varrho e^{-i \varphi} \quad \text { et } \quad p_{2}^{2}=-2(\alpha+\tau r \cos \vartheta) r,
$$

d'où, vu (25),

$$
t=r(\alpha+\tau r \cos \vartheta)[\varrho(\alpha+\varrho \cos \varphi)]^{-1} e^{i \varphi} .
$$

Pour définir $t$, considérons la fonction extrémale $f^{*}(z)$ et la fonction

$$
f_{\delta}^{*}(z)=e^{-i \delta} f^{*}\left(z e^{i \delta}\right) \quad\left(f_{\delta}^{*}(z) \epsilon F_{T}^{\prime}\right),
$$

où $\delta$ est un paramètre qui admet des valeurs quelconques dans le voisinage de zéro.

Considérons en outre la fonction de la variable $\delta$ :

$$
\chi(\delta)=I\left\{f_{\delta}^{*}(r) / a_{1}^{*}\right\}=I\left\{e^{-i \delta} f^{*}\left(r e^{i \delta}\right) / a_{1}^{*}\right\} .
$$

La fonction $\chi(\delta)$ atteint, en vertu de (44), son maximum au point $\delta=0$. Par conséquent

$$
\left(\frac{d}{d \delta} I\left\{\frac{e^{-i \delta} f^{*}\left(r e^{i \delta}\right)}{a_{1}^{*}}\right\}\right)_{\delta=0}=0
$$

Après des calculs aisés on a

$$
\frac{d}{d \delta} I\left\{\frac{e^{-i \delta} f^{*}\left(r e^{i \delta}\right)}{a_{1}^{*}}\right\}=I\left\{\frac{d}{d \delta} \frac{e^{-i \delta} f^{*}\left(r e^{i \delta}\right)}{a_{1}^{*}}\right\}=I\left\{i \frac{r f^{* \prime}\left(r e^{i \delta}\right)-e^{-i \delta} f^{*}\left(r e^{i \delta}\right)}{a_{1}^{*}}\right\},
$$

d'où, vu (45) et en tenant compte de la définition (20), on obtienti

$$
r \tau \cos \vartheta-\varrho \cos \varphi=0 .
$$

En déterminant $r \tau \cos \vartheta$ de (46) et en le substituant dans (43), nous obtenons (après avoir divisé par $a+\varrho \cos \varphi)$,

$$
t=r \varrho^{-1} e^{i \varphi} \text {. }
$$

On obtient done de (28), en vertu de (47),

$$
z_{0}=\omega_{0} e^{-i \delta}
$$

Reprenons maintenant les équations (33) et (34). Nous obtenons à partir de (48), en tenant compte de (41) et (42) et après un calcul facile,

$$
\frac{1}{z_{0}}=\frac{1}{\omega_{0}} e^{i \varphi}=-\frac{A(\varrho, \varphi)}{C(\varrho, \varphi)}-i \frac{B(\varrho, \varphi)}{C(\varrho, \varphi)} .
$$

Par conséquent les équations (35) prendront, selon (49), les formes suivantes:

$$
\begin{aligned}
-\frac{A(\varrho, \varphi)}{C(\varrho, \varphi)} \log \frac{1-\varrho}{1+\varrho}+\log \frac{\varrho}{T\left(1-\varrho^{2}\right)} & =-\frac{A(\varrho, \varphi)}{O(\varrho, \varphi)} \log \frac{1-r}{1+r}+\log \frac{r}{1-r^{2}} \\
-\frac{B(\varrho, \varphi)}{C(\varrho, \varphi)} \log \frac{1-\varrho}{1+\varrho}+\varphi+2 k \pi & =-\frac{B(\varrho, \varphi)}{O(\varrho, \varphi)} \log \frac{1-r}{1+r}
\end{aligned}
$$

d'où, après des calculs aisés on déduit les équations:

$$
\begin{gathered}
\frac{A(\varrho, \varphi)}{C(\varrho, \varphi)} \log \left(\frac{1-\varrho}{1+\varrho}: \frac{1-r}{1+r}\right)-\log \frac{\varrho\left(1-r^{2}\right)}{\operatorname{Tr}\left(1-\varrho^{2}\right)}=0, \\
\varphi+2 k \pi=\frac{B(\varrho, \varphi)}{C(\varrho, \varphi)} \log \left(\frac{1-\varrho}{1+\varrho}: \frac{1-r}{1+r}\right):
\end{gathered}
$$

Remplacons maintenant le système d'équations (50) par un autre système d'équations équivalentes à (50). Posons maintenant pour abréger:

$$
\begin{aligned}
& U(\varrho, r) \equiv \log \{[(1-\varrho) /(1+\varrho)]:[(1-r) /(1+r)]\}, \\
& V(\varrho, r) \equiv \log \left[\varrho\left(1-r^{2}\right) / \operatorname{Tr}\left(1-\varrho^{2}\right)\right] .
\end{aligned}
$$

Les équations (50) prendront alors la forme:

$$
\begin{aligned}
& {[A(\varrho, \varphi) / C(\varrho, \varphi)] U(\varrho, r)=V(\varrho, r),} \\
& {[B(\varrho, \varphi) / C(\varrho, \varphi)] U(\varrho, r)=\varphi+2 k \pi .}
\end{aligned}
$$

De là, vu l'identité évidente $A^{2}(x, y)+B^{2}(x, y) \equiv C^{2}(x, y)$ on a $U^{2}(\varrho, r)=$ $=V^{2}(\varrho, r)+(\varphi+2 k \pi)^{2}$, d'où, supposant $\varphi>0$, on obtient

$$
\varphi=\left[U^{2}(\varrho, r)-V^{2}(\varrho, r)\right]^{1 / 2}+2 k \pi .
$$

En substituant $\varphi$ de (53) dans (51) nous avons avee l'équation (53). un système d'équations équivalentes au système (50) sous condition que $\varphi>0$, ce qui, d'après (52) et vu les inégalités évidentes $C(\varrho, \varphi)>0$, $U(\varrho, r)>0 \quad(\operatorname{car} \varrho<r)$ fait que $B(\varrho, \varphi)>0$ et finalement $\cos \varphi>0$. 
Pour chercher @ il faut alors résoudre l'équation.

$$
\begin{aligned}
{\left[A\left(\varrho,\left[U^{2}(\varrho, r)-V^{2}(\varrho, r)\right]^{1 / 2}\right) / C\left(\varrho,\left[U^{2}(\varrho, r)-\right.\right.\right.} & \left.\left.\left.V^{2}(\varrho, r)\right]^{1 / 2}\right)\right] \times \\
& \times U(\varrho, r)-V(\varrho, r)=0 .
\end{aligned}
$$

Ainsi nous avons démontré la première partie de la démonstration du théorème 1 pour la famille $F_{T^{\prime}}$.

Passons maintenant à la deuxième partie de cette démonstration. Nous démontrons que pour chaque solution $\varrho, \varphi$ des équations (53) et (54), satisfaisant aux conditions: $0<\varrho<r, \sin \left[U^{2}(\varrho, r)-V^{2}(\varrho, r)\right]^{1 / 2}>0$ il existe une for ction univalente $f^{*}(z)$ dans $|z|<1$ de la famille $F_{r}$, telle que $f^{*}(r)=$ $=\varrho e^{i \varphi}$ ou bien $I\left\{f^{*}(r) / a_{1}^{*}\right\}=\varrho \sin \varphi / a_{1}^{*}$.

En choisissant celle pour laquelle le produit $\varrho \sin \varphi$ est le plus grand, nous démontrons la thèse 1 pour les fonctions de la famille $F_{T}$.

Supposons donc que $\varrho, \varphi$ présentent une solution des équations (53) et (54) telle que $0<\varrho<r, \sin \left[U^{2}(\varrho, r)-\nabla^{2}(\varrho, r)\right]^{1 / 2}>0$ et formons pour ces valeurs les expressions correspondantes (41), (42) et (48).

Col sidérons la fonction analytique $H$ qui corresponde à l'integral ${ }^{5}$ ) (55)

$$
\begin{aligned}
\int[\mathfrak{N}(\omega)]^{1 / 2} \omega^{-1} d \omega & =x\left[\bar{z}_{0}^{1 / 2} \log \frac{\varrho e^{-i \varphi} \omega-1+\left[\left(\varrho e^{-i \varphi} \omega-1\right)\left(\varrho e^{-i \varphi} \omega-\varrho^{2}\right)\right]^{1 / 2}}{\varrho e^{-i \varphi} \omega-1-\left[\left(\varrho e^{-i \varphi} \omega-1\right)\left(\varrho e^{-i \varphi} \omega-\varrho^{2}\right)\right]^{1 / 2}}+\right. \\
& \left.+z_{0}^{1 / 2} \log \frac{\varrho e^{i \varphi}\left(1-\varrho e^{-i \varphi} \omega\right)+\left[\varrho e^{i \varphi}\left(1-\varrho e^{-i \varphi} \omega\right)\left(\varrho e^{i \varphi}-\omega\right)\right]^{1 / 2}}{\varrho e^{i \varphi}\left(1-\varrho e^{-i \varphi} \omega\right)-\left[\varrho e^{i \varphi}\left(1-\varrho e^{-i \varphi} \omega\right)\left(\varrho e^{i \varphi}-\omega\right)\right]^{1 / 2}}\right]
\end{aligned}
$$

où $x=\left[2 \varrho^{2} C / T\left(1-\varrho^{2}\right)\right]^{1 / 2}$ et où

$$
\mathfrak{M}(\omega)=-\varkappa^{2}\left(\omega-\omega_{0}\right)^{2}\left(\omega-\varrho e^{i \varphi}\right)^{-1}\left(1-\varrho e^{-i \varphi} \omega\right)^{-1} \bar{z}_{0} e^{-i \varphi} .
$$

Nous remarquons que pour chaque élément $\left\{\hat{\omega}, \hat{M}_{1}(\omega)\right\}$ de $H$ tout autre élément $\left\{\hat{\omega}, \hat{M}_{2}(\omega)\right\}$ du même centre s'exprime par la formule ${ }^{6}$ )

$$
\hat{M}_{2}(\omega)=\varepsilon \hat{M}_{1}(\omega)+2 k \pi i\left(\bar{z}_{2}^{1 / 2}+z_{0}^{1 / 2}\right)+2 l \pi i z_{0}^{1 / 2}
$$

où $\varepsilon= \pm 1$ et $k, l$ sont des nombres entièrs. Nous remarquons encore que pour chaque élément $\left\{\omega^{*}, M^{*}(\omega)\right\}$ de $H$ du centre $\omega^{*}$ situé sur $K^{*}(0,1)$ l'expression $R\left\{M^{*}(\omega)\right\}$ est constante pour $|\omega|=1$, par suite de la définition de $H$ et la propriété concernant la fonction $\mathfrak{N}(\omega)$ sur $K^{*}(0,1)$ (comparer [3], p. 46). Prenons un élément.analytique de $H$ du centre $\omega_{0}$, $\left\{\omega_{0}, M_{0}(\omega)\right\}$ tel que $R\left\{M_{0}\left(\omega_{0}\right)\right\}=0$.

5) On prend ici évidemment leš mêmes branches des racinos $\left(\varrho e^{-i t}(\omega-1)^{1 / 2}\right.$ et $\left[\varrho\left(e^{-i \varphi_{(}}-\varrho\right)\right]^{1 / 2}$ dans le numérateur et le dénominateur.

-) Cela résulte immédiatement de (55).
Il est facile de voir qu'il existe dans l'entourage du point $\omega_{0}$ une courbe regulière $K$ de l'équation

$$
\omega=\omega(s) \quad\left(0 \leqslant s \leqslant s^{*}\right)
$$

et une chaîne d'éléments analytiques,

$$
\{\omega(s), M(\omega, s)\},
$$

de la fonction $H$ le long de cette courbe tels que

$$
\begin{gathered}
|\omega(s)|<1, \quad \omega(0)=\omega_{0}, \quad\left|\omega^{\prime}(s)\right|=1, \\
R\{M[\omega(s), s]\}=0 \quad\left(0 \leqslant s \leqslant s^{*}\right),
\end{gathered}
$$

$$
M^{\prime} \omega[\omega(s), s] \neq 0, \quad\left|M^{\prime} \omega[\omega(s), s]\right|<\infty \quad \text { et } \quad M(\omega, 0)=M_{0}(\omega)
$$

au voisinage de $\omega_{0}$. En effet, prenons comme $K$ la courbe de l'équation (56), vérifiant les conditions (58), passant dans un entourage suffisamment petit du point $\omega_{0}$, issue du point $\omega_{0}$ et telle que $R\left\{M_{0}[\omega(s)]\right\}=0\left(0 \leqslant s \leqslant s^{*}\right)$ Évidemment, vu la condition $M^{\prime}\left(\omega_{0}\right)=0$, une telle courbe existe. En posant en outre $M(\omega, s)=M_{0}(\omega)$, nous obtenons la chaîne démandée.

On voit facilement qu'il existe le plus grand intervalle $\left(0, s^{*}\right)$ de $(56)$ dans lequel existe la courbe $K$, vérifiant les conditions (58). Pour simplifier la désignation nous admettons dans la suite les notations $K$, (56) et (58) comme notations correspondantes à la courbe la plus longue présentée ci-dessus. Remarquons qu'il existe toujours la limite suivante:

$$
\lim _{s \rightarrow s^{*}} \omega(s)=\omega^{*} \quad \text { où } \quad \omega^{*}=0 \quad \text { ou } \quad \omega^{*}=\varrho e^{i \varphi},
$$

ce qui est évident pour $s^{*}$ fini; il suffit alors de le démontrer pour $s^{*}$ infini. En effet, supposant dans ce cas le contraire nous voyons qu'il existe une suite $\left\{s_{n}\right\}$ qui tend vers l'infini et telle que $\omega\left(s_{n}\right) \rightarrow \hat{\omega}$, où $\hat{\omega} \neq 0$ et $\hat{\omega} \neq \varrho e^{i \varphi}$.

Désignons par $\hat{M}(\omega)$ la fonction d'un élément quelconque de la fonction $H$ du centre $\hat{\omega}$. Alors, en posant $M_{n}(\omega)=M\left(\omega, s_{n}\right)$ nous obtenons, pour $n$ suffisamment grand, que

$$
M_{n}(\omega)=\varepsilon_{n} \hat{M}(\omega)+2 \pi k_{n} i\left(\bar{z}_{0}^{1 / 2}+z_{0}^{1 / 2}\right)+2 \pi l_{n} i z_{0}^{1 / 2},
$$

où $\varepsilon_{n}= \pm 1, k_{n}$ et $l_{n}$ sont des nombres entiers donc, vu la rélation évidente $R\left\{M_{n}\left[\omega\left(s_{n}\right)\right]\right\}=0$, que $\varepsilon_{n} R\left\{\hat{M}\left[\omega\left(s_{n}\right)\right]\right\}+l_{n} I\left\{z_{0}^{1 / 2}\right\}=0$ De là, vu $\left.I\left\{z_{0}\right\} \neq 0^{7}\right)$, on a $l_{n}=l=\mathrm{const}$ pour $n$ suffisamment grand. Dans la suite

7) En effet, supposant le contraire on aurait, vu $(49), B(\varrho, \varphi)=0$, et par conséquent, vu (41), $\varphi=\pi / 2$, ce qui est impossible d'après $(50)$.

Annalos Polonici Mathomatici II 
on peut supposer, en choisissant éventuellement la suite partielle de $\left\{s_{n}\right\}$, que $\varepsilon_{n}=\varepsilon=$ const pour $n$ suffisamment grand, d'où

(60)

$$
R\left\{M_{n}(\omega)\right\}=\varepsilon R\{M(\omega)\}+2 \pi l I\left\{z_{0}^{1 / 2}\right\}
$$

pour tout $n$ suffisamment grand.

Désignons par $\hat{K}$ l'are simple de l'équation $\omega=\hat{\omega}(t)$ où $t$ change dans l'intervalle suffisamment petit et dans lequel

$$
\left|\hat{\omega}^{\prime}(t)\right|=1 \quad \text { et } \quad \varepsilon R\{\hat{M}[\omega(t)]\}+2 \pi l I\left\{z_{0}^{1 / 2}\right\}=0 .
$$

Un tel are existe, vu les inégalités $\hat{\omega} \neq \varrho e^{i \varphi}, \hat{\omega} \neq 0$. Du fait que selon (60) les équations

$$
R\left\{M_{n}(\omega)\right\}=0, \quad \varepsilon R\{M(\omega)\}+2 \pi l I\left\{z_{0}^{1 / 2}\right\}=0
$$

sont équivalentes dans un voisinage de $\hat{\omega}$ pour $n$ suffisamment grand, il faut que $\omega\left(s_{n}\right)=\hat{\omega}\left(t_{n}\right)$ pour $n$ suffisamment grand, où $t_{n}$ désigne un certain point de l'intervalle $\langle-\hat{t}, \hat{t}\rangle$. Donc, vu l'équivalence de $(61)$ et $\left|\omega^{\prime}(s)\right|=1$ et $\left|\hat{\omega}^{\prime}(t)\right|=1$, nous obtenons l'identité $\omega(s)=\hat{\omega}\left(s-s_{n}+t_{n}\right)$ dans un voisinage de $s_{n}$. Par conséquent nous avons, en prolongeant, l'identité pour tout $s<s_{n}$ ou $s>s_{n}$ si $t_{n}>0$ ou $t_{n}<0$. Mais cela est impossible, car l'expression $s-s_{n}+t_{n}$ doit être située dans l'intervalle $\langle-\hat{t}, \hat{t}\rangle$, c'est-à-dire l'expression $s-s_{n}$ doit être située dans $\langle-2 \hat{t}, 2 \hat{t}\rangle$ pour chaque $n$, ce qui est impossible. Alors finalement il doit être (59).

Nous distinguons les cas:

a) $\omega^{*} \in K^{*}(0,1)$ et $\omega^{*} \neq \omega_{0}$,

dans lequel nous pouvons évidemment considérer la chaîne (57) dans tout l'intervalle fermé $\left.\left\langle 0, s^{*}\right\rangle^{8}\right)$. Ensuite nous voyons que, pour $s$ proche de $s^{*}$, il faut que

$$
R\{M[\omega(s), s]\}=R\left\{M\left[\omega(s), s^{*}\right]\right\}=0 \quad \text { et } \quad|\omega(s)|<1
$$

et encore $R\left\{M\left(\omega, s^{*}\right)\right\}=0$ pour $|\omega|=1$. Par conséquent il faudrait que $M_{\omega}^{\prime}\left[\omega^{*}\left(s^{*}\right), s^{*}\right]=0$, ce qui n'a pas lieu. Notre cas est donc impossible.

b),$\omega^{*}=\omega_{0}$.

En ce cas, posant $M^{*}(\omega)=M\left(\omega, s^{*}\right)$ on voit que

$$
M^{*}(\omega)=\varepsilon^{*} M_{0}(\omega)+2 \pi k^{*} i\left(\bar{z}_{0}^{1 / 2}+z_{0}^{1 / 2}\right)+2 \pi l^{*} i z_{0}^{1 / 2}
$$

où $\varepsilon^{*}, k^{*}, l^{*}$ sont des nombres analogues à ceux de $(60)$. D'où, pour $\omega=\omega_{0}$, vu les égalités évidentes $R\left\{M_{0}\left(\omega_{0}\right)\right\}=0$ et $R\left\{M^{*}\left(\omega_{0}\right)\right\}=0$ et la condition $I\left\{z_{0}\right\} \neq 0$, nous obtenons que

(63)

$$
l^{*}=0 .
$$

8) C'est possible d'après la rélation (59); la convention pour $8^{*}=\infty$ est évidente.
En outre de (62) on a d'après (63) $R\left\{M^{*}(\omega)\right\}=\varepsilon^{*} R\left\{M_{0}(\omega)\right\}$ dans un voisinage de $\omega_{0}$ et en particulier pour $s$ suffisamment proche de zéro

$$
R\left\{M^{*}\left[\omega\left(s^{*}-s\right)\right]\right\}=\varepsilon^{*} R\left\{M_{0}\left[\omega\left(s^{*}-s\right)\right]\right\}
$$

et finalement $R\left\{M_{0}\left[\omega\left(s^{*}-s\right)\right]\right\}=0$. D'où l'on déduit facilement que $\omega\left(s^{*}-s\right)=\omega(s)$ pour $s$ petits et par conséquent pour chaque $s$. Mais ceci est impossible, car au point $s=s^{*} / 2$ on aurait $\omega^{\prime}(s)=0$, ce qui est contraire à la condition (58). Donc, le cas b) est aussi impossible.

c) $\omega^{*} \in K(0,1)^{\cdots}$ et $\omega^{*} \neq \varrho e^{i_{\varphi}}, \quad \omega^{*} \neq 0$.

Ce cas est impossible, car dans le cas contraire on pourrait selon la condition évidente $M^{* \prime}\left(\omega^{*}\right) \neq 0$ prolonger la courbe $K$ sur l'intervalle plus vaste du paramètre $s$, le nombre $s^{*}$ étant dans notre cas fini d'après (63).

d) $\omega^{*}=\varrho e^{i \varphi}$.

Nous démontrons premièrement qu'il existe un element de $H\left\{\omega^{\circ}\right.$, $M(\omega)\}$ du centre arbitrairement proche du point zéro tel que $R\left\{M^{*}\left(\omega^{*}\right)\right\}=0$. Ceci résulte très facilement du développement de l'integrale (55) dans le voisinage de zéro. Il est facile de deduire qu'il existe pour un tel élément une courbe $K^{*}$ tout à fait analogue à celle de $(56)$ :

$$
\omega=\omega^{*}(s) \quad\left(s_{0} \leqslant s \leqslant s^{* *}\right)
$$

et une chaîne d'éléments analytiques de $H,\left\{\omega^{*}(s), M\left(\omega^{*} ; s\right)\right\}$, le long de cette courbe tels que

$$
\begin{gathered}
\left|\omega^{*}(s)\right|<1, \quad \omega^{*}(0)=\omega^{*}, \quad\left|\omega^{\prime \prime}(s)\right|=1, \\
R\left\{M^{*}\left[\omega^{\prime}(s), s\right]\right\}=0 \quad\left(s_{0} \leqslant s \leqslant s^{*}\right), \\
M_{\omega}^{\prime}\left[\omega^{*}(s), s\right] \neq 0, \quad\left|M_{\omega}^{\prime}\left[\omega^{*}(s), s\right]\right|<\infty \quad \text { et } \quad M^{*}(\omega, 0)=M^{*}(\omega)
\end{gathered}
$$

au voisinage de $\omega$.

Nous remarquons ici qu'il existe dans notre cas les limites

$$
\lim _{s \rightarrow s_{0}} \omega^{*}(s)=\omega_{0}^{*}, \quad \lim _{s \rightarrow 8^{* *}} \omega^{*}(s)=\omega^{* *}
$$

analogues à celles de (59).

Ensuite nous démontrons que les limites (64) ne sont pas égales à zéro simultanément. Dans ce but nous supposons le contraire. Désignons par $G$ l'ensemble $K(0,1)-K$ et considérons dans cet ensemble la fonction analytique, définie dans un voisinage de $\omega^{*}$ par

$$
U(\omega)=M(\omega)-\varepsilon z_{0}^{1 / 2} \log \omega,
$$


où $\varepsilon=+1$ et où $\log \omega$ est une branche de logarithme qui sera définie plus loin. On voit bien de (55) et (30) que $\varepsilon$ peut être choisi d'une façon telle que fonction (65) soit univoque et holomorphe dans $G$. Posons

$$
\omega^{*}(s)=p(s) \exp [i \Omega(s)]
$$

et supposons que la branche de logarithme précédente soit choisie de façon que $\log \omega^{*}(s)=\log p(s)+i \Omega(s)$ pour $s$ suffisamment petit. Dans ce cas nous aurons évidemment selon $(65)$, pour tout $s$,

$$
M^{\cdot}\left[\omega^{*}(s)\right]=U[\omega(s)]+\varepsilon z_{0}^{1 / 2}[\log p(s)+i \Omega(s)] .
$$

En particulier nous obtenons pour $s$ proche de $s_{0}^{*}$ et $s^{* *}$, en posant

$$
\left.U(\omega)=P+i Q+O(\omega)^{9}\right) \quad \text { et } \quad \varepsilon z_{0}^{1 / 2}=\alpha+i \beta
$$

que

$$
\begin{gathered}
R(s)=R\left\{M^{\cdot}\left[\omega^{*}(s)\right]\right\}=P+\alpha \log p(s)-\beta \Omega(s)+O_{1}(\omega) \\
I(s)=I\left\{M^{\cdot}\left[\omega^{*}(s)\right]\right\}=Q+\alpha \Omega+\beta \log p(s)+O_{2}(\omega)
\end{gathered}
$$

et

et alors, selon $R\left\{M^{\cdot}\left[\omega^{\circ}(s)\right]\right\}=0$, nous obtenons

$$
\left.I(s)=\left(\alpha^{2}+\beta^{2}\right) \beta^{-1} \log p(s)+\alpha \beta^{-1} P+Q+O_{3}(\omega)^{10}\right) .
$$

Il en résulte immédiatement que $I(s)$ tend simultanément vers $+\infty$ $($ ou $-\infty)$ pour $s \rightarrow s_{0}$ et $s \rightarrow s^{* *}$, mais cela est impossible, car la fonction $I(s)$ doit évidemment être monotone ${ }^{11}$ ).

Nous démontrons enfin que les courbes $K^{\prime}$ et $K$ n'ont pas de pointis communs. Admettant le contraire, désignons par $\hat{s}$ le plus petit nombre de l'intervalle $\left\langle s_{0}, s^{*}\right\rangle$ pour lequel $\omega^{*}(\hat{s}) \in K$.

On a trois cas:

a) $\omega^{*}(\hat{s})=\omega(s)$, où $0<s<s^{*}$.

Ce cas est impossible, car il existerait les valeurs $s<\hat{s}$ pour lesquelles $\omega^{\circ}(s)$ appartient à $K$.

B) $\omega^{*}(\hat{s})=\omega_{0}$.

Dans ce cas on a $R\{M[\omega, \hat{s}]\}=\varepsilon R\{M(\omega, 0)\}$, et, par conséquent,

$$
R\left\{M^{*}\left[\omega^{*}(s), \hat{s}\right]\right\}=0, \quad R\left\{M\left[\omega^{*}(s), 0\right]\right\}=0,
$$

pour $s$ suffisamment proche de $\hat{s}$ et évidemment $R\{M[\omega(s), 0]\}=0$, pour $s$ suffisamment proche de zéro, ce qui est impossible.

9) $P+i O$ désigne le terme constant du développement de $U(\omega)$ dans un voisinage de $0, O(\omega)$ - la somme d'autres termes d'un rang $\geqslant 1$.

10) $O_{1}(\omega), O_{2}(\omega), O_{3}(\omega)$ d'un rang $\geqslant 1$

11) On en obtient facilement en dérivant la fonction $I(s)$ et en profitant de la condition $R(s)=0$ et des équations de Cauchy-Riemann. $\left.\omega^{*} \hat{s}\right)=\varrho e^{i \varphi}$.

Prenons en ce cas une branche de logarithme définie dans l'entourage du point 1 et $y$ admettant la valeur zéro. Nous voyons facilement qu'on a pour tout $s$

$$
M(\omega, s)=M^{*}(\omega)+2 k \pi i \bar{z}_{0}^{1 / 2}+2 k \pi i z_{0}^{1 / 2}, \quad k=0, \pm 1, \ldots
$$

où $M^{*}(\omega)$ désigne l'intégral (55) dans laquelle les logarithmes se rattachent à la branche choisie ci-dessus. On voit que l'expression $M^{*_{2}}(\omega)$ est univoque dans le voisinage de $\omega^{*}=\varrho e^{i \varphi}$, d'où il résulte immédiatement que

$$
\left[M(\omega, s)-\omega 2 k \pi i \bar{z}_{0}^{1 / 2}-2 k \pi i z_{0}^{1 / 2}\right]^{2}=M^{* 2}(\omega),
$$

pour $s$ suffisamment proche de $s^{*}$.

Analogiquement, nous obtenons que

$$
\left[M^{*}(\omega, s)-2 k \pi i \bar{z}_{0}^{1 / 2}-2 k i \pi i z_{0}^{1 / 2}\right]^{2}=M^{* 2}(\omega), \quad k=0, \pm 1, \ldots
$$

En outre $1 / M^{* 2}(\omega)$ est une fonction holomorphe dans le voisinage de $\omega^{*}=\varrho e^{i_{\varphi}}$, de la derivée différente de zéro.

Ensuite nous aurons, d'aprés $R\{M[\omega(s), s]\}=0, R\left\{M \cdot\left[\omega^{\circ}(s), s\right]\right\}=0$ que $M^{* 2}[\omega(s)]<0, M^{* 2}\left[\omega^{*}(s)\right]<0$, d'où il résulte immédiatement que $\omega\left(s_{0}\right)=\omega^{*}\left(t_{0}\right)$ pour certains $s_{0}<s^{*}$ et $t_{0}<\hat{s}$, ce qui est contraire à la definition de $\hat{s}$.

En résumant, les deux extrémités de la courbe $K$ sont différentes de $\varrho e^{i \varphi}$ et de $\omega_{0}$, un d'eux seulement peurant être égal à zéro. D'autre part, il résulte de la définition de $K^{\circ}$, pareillement comme dans le cas de $K$, que toutes les extrémités de cette courbe doivent être égales à zéro ou à $\omega_{0}$ ou à $\varrho e^{i \varphi}$, ce qui prouve que le cas consideré est impossible.

Finalement il ne reste donc que le cas

e) $\omega^{*}=0$, c'est-à-dire que $\lim _{s \rightarrow s^{*}} \omega(s)=0$.

Après avoir introduit l'are $K$ nous revenons à la démonstration de l'existence de la fonction $f^{*}(z)$, annoncée à la page 192 .

Prenons un arc partiel $C$ quelconque de la courbe $K$ issue du point $\omega_{0}$ et désignons par $\omega=\omega(z)$ la fonction univalente qui transforme le cercle $|z|<1$ en l'ensemble $G=K(0,1)-C$ de façon que $\omega(0)=0$. Supposons encore que l'arc $C$ et la fonction $\omega(z)$ sont choisis de telle façon que $\omega^{\prime}(0)=T e^{i a}$, où $\alpha$ est un nombre réel. Cela est tout à fait possible vu la condition $\lim \omega(s)=0$. On constate très facilement selon la définition de $K$ et de $\stackrel{s \rightarrow s^{*}}{C}$ que l'expression

$$
\mathfrak{M}[\omega(z)] z^{2}{()^{\prime 2}}^{2}(z) / \omega^{2}(z)
$$


est une fonction régulière dans tout le cercle $|z|<1$ et $\mathrm{y}$ possède un seule pôle $\zeta$, tel que $\omega(\zeta)=\varrho e^{i q}$. En choisissant convenablement le nombre precédent $\alpha$ on peut admettre évidemment que $\zeta$ est un nombre réel et positif. De plus l'expression (66) étant, selon la définition de $C$ et de $K$, encore réelle et non négative sur la circonférence $|z|=1$, nous voyons qu'elle doit s'exprimer par la formule

(67) $\quad \mathfrak{M}[\omega(z)] z^{2} \omega^{\prime 2}(z) / \omega^{2}(z)=-\beta \bar{\Delta}(z-\Delta)^{2}(z-\zeta)^{-1}(1-\zeta z)^{-1}$,

où $\beta$ est un nombre positif et $\Delta=e^{i \delta}$ ( $\delta$ réel). De (67) on a

(68) $\{\mathfrak{N}[\omega(z)]\}^{1 / 2} \omega^{\prime}(z) / \omega(z)=(-\beta \bar{\Delta})^{1 / 2}(z-\Delta) z^{-1}(z-\varphi)^{-1}(1-\varphi z)^{-1}$.

Puisque $\omega(0)=0$, il s'ensuit de $(67)$ que

(69)

$$
\Re(0)=\beta \Delta \zeta^{-1} \text {. }
$$

D'autre part

$$
\mathfrak{M}(0)=x^{2} z_{0} \varrho^{-1}
$$

où $x^{2}=2 \varrho^{2} C T^{-1}\left(1-\varrho^{2}\right)$. En comparant $(69)$ et $(70)$ on obtient $\beta / \zeta=x^{2} / \varrho$ $\Delta=z_{0}$. En intégrant les deux membres de (68) et en développant ces intégrales pour $z$ suffisamment petits, nous obtiendrons analogiquement comme à la page 188:

(71) $\quad \bar{z}_{0} \log [(1-\varrho) /(1+\varrho)]+\log 2 \varrho e^{i \varphi}-\log \left[T e^{i \alpha}\left(1-\varrho^{2}\right) / 2\right]+2 k_{1} \pi i$

$$
=\bar{z}_{0} \log [(1-\zeta) /(1+\zeta)]+\log 2 \zeta-\log \left[\left(1-\zeta^{2}\right) / 2\right]+2 k_{2} \pi i,
$$

où $k_{i}=0, \pm 1, \pm 2, \ldots \quad(i=1,2)$. D'autre part, les nombres $\varrho, \varphi$ étant, d'après notre supposition de la page 187 , des solutions des équations (53) et $(54)$, on aura, d'après $(33),(38)$ et $(48)$;

(72) $\quad \bar{z}_{0} \log [(1-\varrho) /(1+\varrho)]+\log 2 \varrho e^{i \varphi}-\log T\left[\left(1-\varrho^{2}\right) / 2\right]+2 k_{1} \pi i$

$$
=\bar{z}_{0} \log [(1-r) /(1+r)]+\log 2 r-\log \left[\left(1-r^{2}\right) / 2\right]+2 \not \dot{k_{2}} \pi i,
$$

où $k_{i}=0, \pm 1, \pm 2, \ldots \quad(i=1,2)$. En comparant les pärties réelles des membres droits de $(71)$ et $(72)$ on obtient, en posant $z_{0}=e^{i \text {, }}$

$$
\begin{aligned}
& \cos v \log [(1-\zeta) /(1+\zeta)]+\log \left[4 \zeta /\left(1-\zeta^{2}\right)\right] \\
& =\cos v \log [(1-r) /(1+r)]+\log \left[4 r /\left(1-r^{2}\right)\right]
\end{aligned}
$$

$$
\zeta=r .
$$

En substituant $r$ au lieu de $\zeta$ dans (71) nous obtenons en nous servant de (72), que $e^{i a}=1$. En résumant, nous voyons que $\omega(z)$ a la forme

$$
\omega(z)=T z+\ldots
$$

Elle appartient donc à la famille $F_{T}$. Puisque nous avons encore, selon (73), que $\omega(\zeta)=\varrho e^{i \varphi}$, donc la fonction (74) satisfait à la condition décrite à la page 192. Le seconde partie du théorème est donc démontrée pour la famille $F_{T}$.

Considérons maintenant la famille $F_{M}$ et la famille des fonctions de la forme

$$
f(z)=F^{\prime}(z) / M_{F}, \quad \text { où } \quad M_{F}=\sup _{|z|<1}|F(z)| \leqslant M .
$$

Les fonctions (75) appartiennent à la famille $F_{T}$, où

$$
T=M^{-1} \text {. }
$$

Désignons comme auparavant par $f^{*}(z)$ la fonction extrémale par rapport à l'opération $K(f)=I\left\{f(r) / a_{1}\right\}$ dans la famille $F_{T}$. Il existe alors dans la famille $F_{M}$ la fonction extrémale $F^{*}(z)$ par rapport à l'opération $K(F)=I\{F(r)\}$, et l'on peut admettre $F^{*}(z)=f^{*}(z) / T$. En posant $F^{*}(r)=$ $=\varrho_{M} e^{i \varphi_{M}}$, on aura selon $(75)$

$$
\varrho_{M}=\varrho M, \quad \varphi_{M}=\varphi,
$$

où $\varrho$ et $\varphi$ gardent leur significations antérieures. En substituant $\varrho$ et $\varphi$, fournis par (77), et $T$ fourni par (76) dans (53) et (54), nous obtenons les équations (4). C'est ce qu'il fallait démontrer.

Démonstration du théorème 2. Dans ce but nous traiterons pour le moment $M$ comme variable et passerons à la limite dans les équations (4), en faisant tendre $M$ vers l'infini. Puisque $\varrho_{M}$ est borné (voir [1], p. 89), on obtient de (4) que $\varrho_{M}$ et $\varphi_{M}$ ont des limites $\varrho_{\infty}$ et $\varphi_{\infty}$ satisfaisant aux équations

$$
\begin{aligned}
\log \varrho_{\infty} & =\log \left[r /\left(1-r^{2}\right)\right]+\sin \varphi_{\infty} \log [(1+r) /(1-r)], \\
\varphi_{\infty} & =\cos \varphi_{\infty} \log [(1+r) /(1-r)] .
\end{aligned}
$$

De même, en désignant par $S(r, M)$ le produit $\varrho_{M} \sin \varphi_{M}$, on a $S(r, M)$ $\leqslant S(r, \infty)$, car $S(r, M)$ est la fonction monotonique de $M$.

Considérons maintenant la famille $F_{\infty}$. Soit $F \in F_{\infty}$ une fonction quelconque de cette famille. Considérons la fonction auxiliaire

$$
\Phi_{x}(z)=\varkappa^{-1} F(\varkappa z)=z+A_{2} \varkappa z^{2}+\ldots,
$$

définie dans le cercle $|z|<x<1$ et univalente. La fonction (79) est bornée, soit $\sup _{|z|<1}\left|\Phi_{*}(z)\right|=M_{x}, z=r$ étant fixé, on a évidemment

$$
I\left\{\Phi_{x}(r)\right\} \leqslant S\left(r, M_{\varkappa}\right) \leqslant S(r, \infty) .
$$


D'autre part $I\left\{\Phi_{x}(r)\right\} \rightarrow I\left\{F^{\prime}(r)\right\}$ si $x \rightarrow 1$, donc finalement on obtient de (78) et (80) évaluation de la partie imaginaire de la fonction $F(z)$ dans $F_{\infty}$ de la forme $\left.I\left\{F^{\prime}(r)\right\} \leqslant S(r, \infty)^{12}\right)$.

Pour démontrer que la limite $S(r, \infty)$ est atteinte considérons maintenant la suite de fonctions extrémales $F_{n}^{*}(z)$ par rapport à l'opération $K(F)$ dans la famille $F_{M_{n}}$, où $M_{n}$ est une suite convergente vers l'infini. On peut admettre, vu que la famille $F_{\infty}$ est normale, par le choix d'une suite partielle, que la suite $F_{n}^{*}(z)$ est convergente vers une certaine fonction limite $F_{0}(z)$. On a alors

$$
\lim I\left\{F_{n}^{*}(r)\right\}=I\left\{F_{0}(r)\right\},
$$

et comme $I\left\{F_{n}^{*}(r)\right\}=S\left(r, M_{n}\right)$ on a $I\left\{F_{0}(r)\right\}=S(r, \infty)$, ce qui démontre finalement le théorème 2.

\section{Travaux cités} 194

[1] L. Bieberbach, Lehrbuch der Funktionentheorie, vol. I et II, New York

[2] Z. Charzyński, Sur les fonctions univalentes bornées, Rozprawy Mate. matyczne $2(1953)$, p. 3.57 .

[3] Z. Charzyński, W. Janowski, Sur l'équation générale des fonctions extrémales dans la famille des fonctions univalentes bornées, Annales Universitatis Mariae Curie-Skłodowska 4, Sectio A, Mathematica (1950), p. 41-56.

4] H. Grunsky, Neue Abschätzungen zur Konformen Abbildung ein- und mehrfach zusammenhängender Bereiche, Math. Sem. Univ. Berlin 1 (1932).

12) Les résultats obtenus pour les fonctions non bornées coïncident avec ceux que H. Grunsky a obtenu par d'autres procédés; voir [4].

\section{Sur les fonctions univalentes $K$-symétriques}

\author{
par W. JANOWskI (Éódź)
}

Considérons les fonctions holomorphes univalentes $K$-symétriques dans le cercle $|z|<1$ de la forme

$$
\Phi(z)=z+B_{2} z^{K+1}+B_{3} z^{2 K+1}+\ldots,
$$

où $K$ est un nombre entier et positif. Soit $M>1$ un nombre positif quelconque, $\Phi_{M}$ - la famille de toutes les fonctions bornées de la forme (1) assujetties à la condition $|\Phi(z)|<M$ et $\Phi_{\infty}-$ la famille de toutes les fonctions de la forme (1).

Les valeurs $z$ ou bien $1>r>0$ étant fixées, on peut s'occuper des expressions suivantes
(2) $|\Phi(z)|$,
(4) $R\{\Phi(r)\}$,
(3) $\left.\quad \arg (\Phi(z) / z)^{1}\right)$,
(5) $\quad I\{\Phi(r)\}$

au sens de trouver leurs limites supérieures dans les familles $\Phi_{M}$ et $\Phi_{\infty}$.

On voit bien que pour les expressions (2)-(4) on obtient ces limites immédiatement, en profitant des résultats connus pour les fonctions 1-symétriques (voir [2] et [4]).

Pour les fonctions de la famille $\Phi_{M}$ et respectivement pour les fonctions de la famille $\Phi_{\infty}$ on a des inégalités

$|\Phi(z)| \leqslant R_{M}, \quad \Phi(z) \in \Phi_{M}$,

ò̀ $R_{M}\left(0<R_{M}<|z|\right)$ satisfait à l'équation $R_{M}^{K} /\left[1-\left(R_{M} / M\right)^{K}\right]^{2}=|z|^{K} /\left(1-|z|^{K}\right)^{2}$;

oì $R_{\infty}=|z| /\left(1-|z|^{K}\right)^{2 / K}$.

(8)

$|\Phi(z)| \leqslant R_{\infty}, \quad \Phi(z) \in \Phi_{\infty}$

$\arg (\Phi(z) / z) \leqslant \Omega_{M}, \quad \Phi(z) \in \Phi_{\infty}$

où $\Omega_{M}$ et $R_{M}\left(0<R_{M}<|z|\right)$ satisfont aux équations

1) On prend la branche de l'argument qui est égale à 0 , pour $z=0$. 